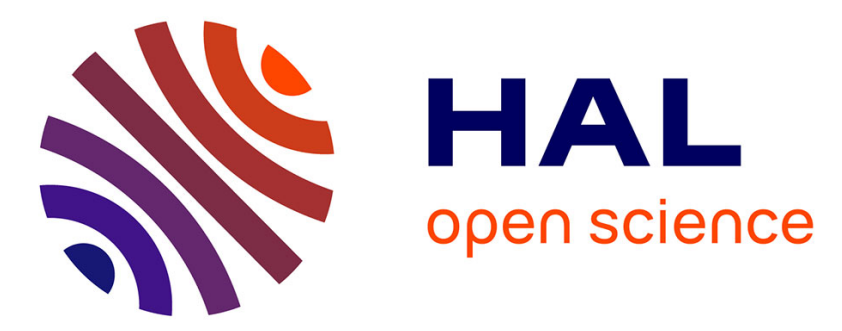

\title{
L'a-llusion. Il était perdu mais il ne le savait pas
}

Michèle Benhaim

\section{To cite this version:}

Michèle Benhaim. L'a-llusion. Il était perdu mais il ne le savait pas. Figures de la psychanalyse, 2011, 10.3917/fp.022.0055 . hal-01429363

\section{HAL Id: hal-01429363 https://hal-amu.archives-ouvertes.fr/hal-01429363}

Submitted on 7 Jan 2017

HAL is a multi-disciplinary open access archive for the deposit and dissemination of scientific research documents, whether they are published or not. The documents may come from teaching and research institutions in France or abroad, or from public or private research centers.
L'archive ouverte pluridisciplinaire HAL, est destinée au dépôt et à la diffusion de documents scientifiques de niveau recherche, publiés ou non, émanant des établissements d'enseignement et de recherche français ou étrangers, des laboratoires publics ou privés. 


\title{
L'A-LLUSION. IL ÉTAIT PERDU MAIS NE LE SAVAIT PAS...
}

Michèle Benhaim

\author{
ERES | « Figures de la psychanalyse »
}

$2011 / 2 n^{\circ} 22 \mid$ pages 55 à 65

ISSN 1623-3883

ISBN 9782749214924

Article disponible en ligne à l'adresse :

http://www.cairn.info/revue-figures-de-la-psy-2011-2-page-55.htm

\section{Pour citer cet article :}

Michèle Benhaim, «L'a-llusion. Il était perdu mais ne le savait pas... », Figures de la psychanalyse 2011/2 ( $\left.\mathrm{n}^{\circ} 22\right)$, p. 55-65.

DOI 10.3917/fp.022.0055

Distribution électronique Cairn.info pour ERES.

(c) ERES. Tous droits réservés pour tous pays.

La reproduction ou représentation de cet article, notamment par photocopie, n'est autorisée que dans les limites des conditions générales d'utilisation du site ou, le cas échéant, des conditions générales de la licence souscrite par votre établissement. Toute autre reproduction ou représentation, en tout ou partie, sous quelque forme et de quelque manière que ce soit, est interdite sauf accord préalable et écrit de l'éditeur, en dehors des cas prévus par la législation en vigueur en France. Il est précisé que son stockage dans une base de données est également interdit. 


\section{L'a-llusion. Il était perdu mais ne le savait pas...}

- Michèle Benhaim •

Nous nous sommes ${ }^{1}$, du point de vue psychanalytique, penchés sur les effets du désir maternel sur l'enfant, sur la construction de son fantasme et de son sinthome ${ }^{2}$, sur la portée du fantasme maternel sur la structure de l'enfant, sur les dimensions symptomatiques de son organisation subjective. Nous savons que le dialogue mère/bébé demeure fondamental et s'inaugure d'emblée sous le premier regard posé, échangé, au détour des premiers mots signifiant à l'enfant le lieu d'inscription historique qui sera le sien, premiers mots qui, dans certains cas, retentiront toute une vie, lors de la mythique première tétée et du désir alors engagé par l'une, la mère, et mis en mouvement chez l'autre, le bébé.

Le bébé, du fond de sa détresse primordiale ${ }^{3}$, peut affirmer ou refuser son absolue dépendance ${ }^{4}$ à l'égard de son prochain secourable ${ }^{5}$... Nous connaissons le sort qui peut être celui d'un désir inconscient maternel meurtri et l'épuisement psychique qui peut, en écho, gagner, voire décourager, temporairement ou à

Michèle Benhaim, professeur de psychopathologie clinique, université de Provence, Laboratoire de psychanalyse et psychopathologie clinique, EA 32 78, psychanalyste à Marseille.

1 M. Benhaïm, 1990, La folie des mères, Paris, Imago, réed. 2008 et 2001, L'ambivalence de la mère, Toulouse, érès, coll. " Poche ", 2011.

2 Sinthome : ce qui noue et fait tenir ensemble RSI afin de se confronter sans trop de dommages à la réalité (J. Lacan, Le Séminaire, Livre XXIII [1975-1976], Le sinthome, Paris, Le Seuil, 2005).

3. S. Freud, 1895, "Esquisse pour une psychologie scientifique ", dans La naissance de la psychanalyse, Lettres à Wilhelm Fliess 1887-1904, Paris, PUF, 2006.

4. D. W. Winnicott, 1970, «Le passage de la dépendance à l'indépendance dans le développement de l'individu ", dans Processus de maturation chez l'enfant, Paris, Payot.

5. S. Freud, 1895, "Esquisse pour une psychologie scientifique », op. cit. 
jamais, le bébé dans ses tentatives de " relance $^{6}$ » de la Préoccupation Primaire de sa mère.

Au cœur d'une clinique adolescente sous transfert, il nous a été donné de vérifier l'état de mort (deadness ${ }^{7}$ ) du monde intérieur de la mère, en l'occurrence au détour de la réactivation de la dépression maternelle chez l'adolescent(e) ; le travail a consisté en une élaboration pour le sujet adolescent pris dans les rets de la con-fusion, d'atteindre sa propre culpabilité et ses angoisses dépressives singulières.

Les énigmes actuelles de cette clinique adolescente qui témoigne d'absences d'inscriptions expérientielles et/ou historicisantes, de mises en actes répétitives et qui ne cessent pas de ne RIEN écrire, nous poussent à interroger ce qu'il en est du maternel aujourd'hui.

Nous allons questionner ici ce qu'il en est du rapport maternel au « désir » qui tenterait de se passer de la limite et donc d'écrire de l'illimité, autrement dit d'une impossibilité maternelle à " illusionner " son enfant, ou encore d'un " désir " qui ferait sa $\operatorname{loi}^{8}$ au creux d'une jouissance immanquable...

Pour ce faire, renversons la logique pathogène communément admise qui veut qu'à ne pouvoir renoncer à l'enfant-phallus, une mère ferme la porte à toute velléité désirante chez son enfant, le maintenant silencieux, voire mutique, ou encore, captif d'une désillusion impossible.

Et si une des figures de la jouissance maternelle empruntait la voix quasi inverse ? Développons : un fait de structure reposant sur une exigence éthique humaine convoque la mère à se présentifier à l'enfant comme un $\mathrm{A}$. Ce temps logique recouvre, nous semble-t-il, le temps de la désillusion chez Winnicott, cette épreuve maternelle qui consiste à signifier à l'enfant qu'il n'est ni omnipotent, ni tout-puissant, autrement dit, en termes kleiniens, à lui ouvrir l'accès à la position dépressive salvatrice en ce qu'elle permet à l'enfant de s'extraire du champ psychotique de l'indifférenciation. Si A, alors $\$$...

La désillusion suppose un temps logique antérieur, celui de l'illusion, comment perdre en effet, si l'on n'a rien à perdre ? Ce temps de l'illusion pour-

6. J. Bergès et G. Balbo, Jeu des places de la mère et de l'enfant. Essai sur le transitivisme, Toulouse, érès, 1998.

7. D. W. Winnicott, "La réparation en fonction de la défense maternelle organisée contre la dépression ", dans De la pédiatrie à la psychanalyse, Paris, Payot, 1948.

8. G. Morel, La loi de la mère, Paris, Economica, 2008. 
rait recouvrir un temps mythique où le sujet n'est pas (encore) barré (S). «..., et $S$, [c'est] le sujet encore non existant, qui a à se situer comme déterminé par le signifiant $^{9} "$.

Si l'on admet l'extrême nécessité de la désillusion, force est d'admettre la non moins nécessaire illusion. Nous avancerons que pour illusionner l'enfant, la mère " doit » se présentifier à lui comme non castrée $(A)$, c'est-à-dire dans une jouissance "illimitée ". Tout en sachant, elle, que c'est une illusion, elle en passe par une sorte de déni cadré du manque, mécanisme que lui ouvrent les bouleversements psychiques déclenchés par le temps de la grossesse, ce temps où le principe de plaisir semble prendre le dessus sur le principe de réalité, directement suivi et donc encore un peu entretenu par « la maladie normale » que Winnicott nomme Préoccupation Maternelle Primaire.

Continuons de poser les éléments d'un cadre propice à l'accueil de notre hypothèse : la mère réelle recouvre ce que Freud nomme "La Chose "; or, la Chose possède cette particularité de désigner la jouissance comme relevant d'un impossible en même temps qu'elle en est, osons-le, le " signifiant ". " Le désir vient de l'Autre et la jouissance est du côté de la Chose ${ }^{10}$. »

L'hypothèse relative à la jouissance maternelle post-moderne recelée dans une clinique adolescente de l'acte est la suivante : la mère pourrait bien, en se présentifiant comme d'ores et déjà barrée, empêcher, en ne pouvant investir libidinalement son enfant, le temps de l'illusion et donc celui de la désillusion. La conséquence de ce défaut d'un instant logique auquel vient se substituer le moment de conclure produit un sujet ayant à exister comme divisé sans avoir pris acte de la perte qui le fonde, c'est-à-dire sans avoir traversé le temps pour comprendre. Comme si sa vie commençait par la conclusion... Le sujet existe ici avec un objet perdu, mais dont la perte ne repose pas sur le mythe d'une complétude antérieure : I'objet n'aurait jamais été à perdre et le sujet serait né d'ores et déjà divisé. Or, le psychisme se fonde d'une perte (logique). Chez les adolescents à l'origine de cette hypothèse, l'objet ne semble pas être à perdre, du "vide " se crée du fait que le sujet semble évoluer sans aucun rapport à la qualité perdue de l'objet. Cette clinique témoigne d'un retour dans le réel de ce temps escamoté, notamment dans la toxicomanie, où l'illusion ne cesse d'être recherchée,

9. J. Lacan, Le Séminaire, Livre X (1962-1963), L'angoisse, Paris, Le Seuil, 2004, p. 37.

10. J. Lacan, Écrits, Paris, Le Seuil, 1966. 
les conduites ayant à voir avec le vide, la mort... chez de jeunes patients paraissant souffrir de la persistance de traits dépressifs, mais qui l'ignorent.

En écho, qu'est-ce qu'une mère trop immédiatement barrée ? Peut-être cette figure que Green ${ }^{11}$ dépeint sous les traits d'une " mère morte », figure réactivée toutes les fois où l'adolescent, ayant subi un non-investissement maternel trop massif, va tenter de combler le vide psychique.

Un bébé qui n'aurait pas été investi libidinalement serait ce bébé auquel la mère n'autorise pas la croyance en sa toute-puissance, ce qui le confronterait, dans ce temps d'un narcissisme non encore solidement établi, au vide, ce vide de signifiant, sorte de réel " absolu » qui fait retour à l'adolescence, à l'occasion de la traversée du réel pubertaire. En termes freudiens, c'est un bébé que la mère n'aurait pas séduit, l'empêchant de la sorte à séduire en retour, tout en entrevoyant, dans cette première séductrice qu'est la mère, l'objet cause de son désir, autrement dit l'objet.

Les apparences sont trompeuses : il ne s'agit pas ici d'adolescents ne « supportant pas la frustration ", c'est-à-dire ce rappel de la désillusion, comme nous pouvons l'entendre dire dans nombre d'institutions socio-éducatives, mais de jeunes dont les actes plus que les symptômes témoignent d'un temps qui fait défaut, celui de l'illusion. Paradoxalement, de n'avoir pas bu la coupe de l'omnipotence sur le monde en temps voulu, certains adolescents manifestent une sorte de toute-puissance incessante et inentamable au travers d'un langage insultant ou de passages à l'acte violents, comme identifiés à un objet d'ores et déjà déchu et non à un objet $a$, à choir, signification incarnée dans le réel du discours de la mère, enfant pris dans le désir de l'Autre.

Aussi difficile que soit, avec certains de ces jeunes, l'établissement du transfert, les rencontrer au sens plein fonctionne comme un coup de vent pouvant les effondrer. La rencontre, comme entaillant le narcissisme aux contours fragiles, assiste à une sorte de dépressivité adolescente qui fera socle, sous transfert, à un processus de perte envisageable. Ce qui montre bien que, contrairement aux apparences manifestes, l'adolescent tente de croire (par ses mises en acte illimitées) à une toute-puissance, certes, mais parce que justement il n'y a jamais cru, il n'y a jamais eu accès. Et la toute-puissance, insistons-y, recouvre le narcissisme freudien, l'illusion winnicottienne, et le bébé dont Lacan fera d'abord « un pur être de jouissance ».

11. A. Green, Narcissisme de vie, narcissisme de mort, Paris, Minuit, 1983. 
On l'aura compris, ce temps de la toute-puissance est nécessaire au nourrisson et demeure possible d'accès si la mère s'offre d'abord comme A non barrée. Il va de soi que c'est d'un temps mythique et non chronologique dont il est question, même si Winnicott l'intitule Préoccupation Maternelle Primaire, préoccupation dont il ajoute qu'elle repose sur " un environnement parfait ${ }^{12}$ » (1949), c'est-àdire un environnement qui s'adapte activement aux besoins de ce nouveau psyché-soma qu'est le nourrisson à la naissance.

Si le réel fait retour à l'adolescence, nous pouvons supposer que c'est en raison de la découverte qui s'impose au sujet lors de ce passage : l'adolescent découvre en effet que sa mère est une femme ${ }^{13}$, autrement dit, se réactualise pour lui un $\mathrm{A}$ à barrer, un instant de (re)voir. Si c'est bien d'une réédition dont il s'agit alors, l'adolescent pourra supporter cette découverte sans sombrer dans un désespoir trop violent, c'est-à-dire en traversant juste un moment de " crise ". À l'inverse, si c'est bien d'un temps inaugural du voir dont il est question, temps auquel, rappelons-le, se serait substitué dans les premiers instants de la vie le moment de conclure sans comprendre, alors le champ s'ouvre aux manifestations pathologiques exacerbées que sont toutes les transgressions dangereuses.

De quel processus procède dans ces cas la jouissance de la mère ? Peut-être justement a-t-elle consisté à ne pas illusionner le bébé, à se présenter d'emblée comme A, à faire fi d'un temps logique fondateur de la subjectivité qui consiste à se débrouiller tous deux, mère et bébé, de la perte fondamentale sur laquelle le désir de l'une va se remettre en mouvement du côté de la femme, non pas contre, mais avec la mère (" devenir mère, c'est perdre la féminité ", dit Lucien Israël ${ }^{14}$ ) et le désir de l'autre, le bébé, va émerger dans l'altérité, condition de la subjectivité.

À défaut, la mère peut apparaître dans une course folle à la jouissance infinie, consommant son enfant au lieu d'en faire un phallus. C'est peut-être ce que contiennent ces propos terribles d'une mère au thérapeute de son fils de 2 ans

\footnotetext{
12. "L'environnement parfait est celui qui s'adapte activement aux besoins du psychésoma nouvellement constitué, ce que nous, observateurs, savons être le nourrisson à ses débuts. Un environnement défectueux est mauvais parce que, par défaut d'adaptation, il empiète sur le psyché-soma (c'est-à-dire le nourrisson) qui est forcé de réagir. De ce fait, il y a perturbation de la continuité d'existence du nouvel individu. "

13. M. Benhaïm, "La mère est-elle sexuée ? ", dans De l'infantile au juvénile, Toulouse, érès, coll. « Le Bachelier », 2006.

14. L. Israël, La jouissance de I'hystérique, Paris, Le Seuil, coll. « Points », 1974.
} 
atteint d'une maladie somatique grave, propos énoncés en présence de l'enfant : "Cet enfant-là, s'il n'était pas là, il ne nous manquerait pas... " Or, un enfant, d'être le phallus, c'est-à-dire un symbole " moins ", ça manque, même quand c'est là... L'avenir dira combien ou comment cet enfant se constituera ou pas prisonnier de ce discours maternel emprunt d'un excès de présence toute-puissante sur l'enfant, reflet de la jouissance mortifère de sa mère, s'il s'échouera au champ de l'Autre ou s'il s'en délogera.

D'emblée et encore petit enfant, le sujet est confronté à la jouissance de sa mère, il lui est nécessairement assujetti, ce qui signe son inscription dans son désir et il va s'en extraire en en conservant, au mieux des traces, au pire en réalisant le vœu maternel inconscient. Les obstacles du processus maternel reposent sur le paradoxe qui prescrit qu'avoir un enfant empreinte phalliquement une femme tout en rappelant la castration à son bon souvenir : avoir un enfant, c'est justement ne plus l'avoir. Si reconnaître que la mère est une femme s'avère nécessaire à la structuration subjective, ne l'entrevoir que comme femme fait obstacle au processus d'intégration de la perte : décompléter la mère, pour un enfant, suppose de l'avoir auparavant comblée et occuper cette position, pour une femme, suppose de se détourner, un temps, de la "féminité ", un temps seulement, insiste Winnicott qui octroie quelques mois d'existence à la dévotion maternelle, un temps seulement si I'on se souvient, avec Freud, que le seul rapport sexuel ne saurait être qu'incestueux ou meurtrier. La mère n'est ni toute, ni tout pour l'enfant. Pourtant, le désir de la mère réelle, la mère primordiale, est un désir incestueux qui fonde la structure si, cadré d'être illusoire, il ne bascule pas dans le crime.

Ce désir d'inceste (réintégration de son produit) relève d'un temps logique nécessaire en ce qu'il demeure " désir » causé par un objet perdu et, qu'en cela, il représente pour la première fois le manque réel.

À cette condition, l'enfant fera de cette mère réelle, sa " maman ", une mère imaginaire et symbolique $(A)$, répondant ainsi à sa demande, celle d'être reconnue mère par son enfant, en même temps que son propre statut d'enfant imaginaire et symbolique (l'enfant conçu, porté, attendu, fantasmé, etc.) deviendra réel, puisqu'il sera un autre non confondu avec le corps de sa mère. L'amour reposera sur cette perte pour la mère de ce bout de corps réel et produira ces renversements. Nous pouvons penser que l'économie de ces derniers peut avoir pour effet d'abolir la dialectisation de la castration fondatrice d'un lien entre l'un et l'Autre et les laisser chacun à la solitude de leur division. 
Ainsi, de la même façon qu'une mère captive d'une jouissance absolue, c'està-dire meurtrière, ne se confrontera, pas plus qu'elle ne confrontera l'enfant, au manque, une mère d'ores et déjà divisée n'a aucun interdit à énoncer et sur ce défaut se nouent les défaillances symboliques repérables dans l'utilisation du langage, le rapport à la parole et les passages à l'acte de certains adolescents qui livrent l'autre à leur caprice comme, sans doute, eux-mêmes ont été offerts aux caprices de l'Autre Réel. Pour qu'il y ait castration, encore faut-il de l'Autre à castrer. Encore une fois, la maternité ne se décline pas en termes de "la femme "contre" la mère ${ }^{15}$ ", mais bien de la fonction maternelle " avec " la position féminine.

L'enfant, lui, est dans cette nécessité d'avoir été un temps un objet réel pour, à partir de ce statut, construire un fantasme, c'est-à-dire la clé de son destin. G. Morel ${ }^{16}$ développe largement combien " en même temps que notre mère nous parlait, nous avons plongé les racines de notre désir dans le sien " et aussi comment « toute notre vie nous portons la marque de son désir et les stigmates de sa jouissance ".

Se défaire de la jouissance de la mère relève d'une opération psychique nécessaire, la séparation. Pierre Bruno propose une très juste définition du sujet en rapport à cette nécessité de se construire contre la jouissance de la mère : «Que suis-je ? Une résistance. [...] Sa capacité, aussi précaire soit-elle, à user de signes qui se déposent dans la nature, mais encore et surtout faut-il voir dans cette résistance qu'il est, y compris à être sujet, le cœur même du symptôme. Cette résistance n'est pas seulement en effet refus à l'Autre, mais refus fécond à soi, en tant qu'inclus dans l'Autre par le biais de l'assujettissement ${ }^{17}$. " Nous savons que cet opérateur au fondement de toute subjectivité, la séparation, suppose un pendant que Lacan nomme " aliénation ».

Les deux processus bien en place ouvrent le champ de la structure névrotique, I'aliénation seule celui de la psychose, l'en deçà de l'aliénation celui des autismes ${ }^{18}$. Que serait une séparation seule, si ce n'est le point d'origine du vide, sortes d'états du sujet actuels où le vide en place de manque caractérise certains

15. M. Zafiropoulos, La question féminine, de Freud à Lacan, Paris, PUf, 2010.

16. G. Morel, La loi de la mère, Paris, Economica, 2008.

17. P. Bruno, "Changement de psychanalyse ", Psychanalyse, $n^{\circ} 1$, Toulouse, érès, 2004.

18. C. Soler, L'inconscient à ciel ouvert dans la psychose, Toulouse, PUM, 2008. 
mouvements adolescents ? Difficile de se repérer en termes binaires (nom du père ou forclusion) face à certains de ces mouvements presqu'asymptomatiques. Le vide semble exclure l'élaboration d'un symptôme et se résoudre dans le passage à l'acte comme autant de tentatives répétées de s'accrocher aux parois du vide afin d'éviter de sombrer aux tréfonds du réel.

La clinique de la névrose nous décline des symptômes que l'analyse synthétisera en un sinthome figuré en général par un nom du père, la " transformation de la misère hystérique en malheur banal », disait Freud en 1893.

À partir de suppléances psychotiques, s'édifie le sinthome figuré par autre chose qu'un nom du père.

La clinique adolescente actuelle nous confronte parfois d'abord à ce vide qu'il va falloir, dans un premier temps, border, identifier, désigner comme ayant la possibilité d'un bord. Supposons que ce vide (de la pensée, de l'inscription, de I'historicité, de la parole équivalente à la non-parole, "Je dis ça, je dis rien " aiment à souligner certains adolescents, de l'acte dont ils ne voient pas la portée ou l'incidence ...) s'origine de ce temps du « désaide » où le sujet se débat dans un univers chaotique qu'un " prochain secourable " se doit d'organiser et qu'en ce temps, cet Autre organisateur tout-puissant ne lie pas la première satisfaction au premier déplaisir. Voilà qui a-ssigne le sujet, sujet alors non-signé de la jouissance maternelle, si ce n'est celle de lui en proscrire l'accès d'emblée, c'est-à-dire de ne pas l'illusionner.

Lacan, dans L'envers de la psychanalyse, souligne deux destins à la question de l'inscription d'un sujet dans l'interprétation de la jouissance maternelle : l'énigme, dans la névrose où cette jouissance fait question pour l'enfant sous la forme d'énoncés tels que : "Que me veut-elle ? ", "Que (qui) suis-je pour elle ? ", "Que va-t-elle bien chercher ailleurs alors que je suis là ? ", etc. ; la citation, sorte de réponse sans question qui bouche le manque dans la psychose, certitude partagée par la mère et l'enfant d'un savoir absolu de la première sur le second ; pourrions-nous situer à mi-chemin " l'a-ssignation " ? La place dévolue à l'enfant serait celle d'un objet pur laissé tombé dans un vide, sans bords sur lesquels inscrire quelque interprétation du désir maternel que ce soit, interprétation sur laquelle repose pour chacun le nœud symptomatique/sinthomatique.

L'écriture de la métaphore paternelle fait place à deux endroits (stratégiques, certes, de s'annuler) au Désir de la Mère (DM). Le destin névrotique consiste à être délogé d'une position d'objet de la mère par un (Nom Du) Père. L'impasse psychotique témoigne d'un impossible de cette opération métaphorique. L'a- 
ssignation voit-elle le sujet immédiatement expulsé de sa position d'objet, c'està-dire non d'abord investi par le désir de la mère ? Certains adolescents semblent y avoir consenti...

Être " assujetti " au désir de la mère, si le refoulement barre la jouissance primordiale de la mère, produit la rencontre avec le manque et ne saurait se confondre avec " être a-ssigné » par la jouissance maternelle qui produit la rencontre avec le vide. Qu'en est-il alors, en effet, des premiers liens mère/enfant dont on sait combien ils gravent inéluctablement le destin pulsionnel et libidinal du sujet?

Un temps initial « érotique » avec la mère est indispensable à la survie corporelle et psychique de l'enfant, " investi libidinalement », dit Freud. Mais ce temps, d'être illusoire, s'arrête, dit Winnicott. Ces deux temps logiques s'originent des filtres inconscients à l'œuvre dans les soins maternels. Si la condition du désir du sujet en passe par un renoncement, encore faut-il qu'il y ait de quoi renoncer.

Quel sort est fait au désir inconscient maternel aujourd'hui ? Désir inconscient devant s'énoncer comme le désir d'avoir un enfant à tout prix (Procréations Médicalement Assistées, nombre croissant de consultations psy de femmes de 40-45 ans ne parvenant pas à être enceintes...), désir s'énonçant, de plus, dans un discours social que l'on peut qualifier de « bipolaire ", mélancolico-pervers, où les nouveaux destins de certains nourrissons (congélation, combustion...) témoignent dans le réel d'enfants conçus dans une sorte de logique impersonnelle où I'on fabrique un objet plus qu'on ne le conçoit. Concevoir au sens de porter l'enfant dans son corps et dans sa psyché ${ }^{19}$, c'est-à-dire au sens de l'engagement d'un sujet (mère) qui, au nom d'un " père ", promet son désir dans un processus (pro)créateur et non dans un comportement automatisé au sein d'une usine hypertechnicisée.

La logique sociale hyperscientifique actuelle n'épargne pas les processus parentaux et les ampute de leur dimension subjective, comme elle ampute les soins de leur dimension transférentielle ou l'éducation de sa dimension ludique.

Si le sujet est impliqué différemment dans le processus de (pro)création, alors attendons-nous à une modification de plus en plus profonde du statut de l'objet (enfant) et étonnons-nous des méandres que cet objet va parcourir pour espérer émerger dans sa singularité, engagé possiblement dans un vivre ensemble, c'est-

19. M. Benhaïm, La folie des mères, op. cit. 
à-dire dans une altérité, sachant que, là où la jouissance est pleine, I'altérité laisse peu ou pas de traces.

À quel désir de l'Autre se vouer quand l'Autre tient ce discours annihilant toute dimension désirante, quand une mère, du fond de sa dépression et/ou d'une dépressivité ambiante, envisage la venue de cet autre qu'est l'enfant comme un " excès ", quand une mère délaissée n'offre plus comme lieu d'accueil à l'enfant qu'un imaginaire trop douloureux, trop endeuillé, c'est-à-dire un espace où son objet (l'enfant) se verra soit consommé, soit englué dans cette douleur qu'il va s'acharner, voire s'épuiser à tenter de déplacer et que signent ces passages à l'acte à répétition chez certains adolescents, comme autant de tentatives d'appels échoués en cris sourds ?

Un enfant à aimer doit d'abord avoir été un bébé à croquer... parce qu'« aimer, c'est dévorer ${ }^{20}$ ", sauf que la mère inscrit d'emblée cet amour dévorant dans un processus ludique de semblant. Ce qui permet cet écart et sauve en général l'enfant d'être victime d'infanticide, c'est-à-dire de la " gueule du crocodile ${ }^{21}$ ", c'est la position féminine, non pas contre, encore une fois, mais avec la mère.

Comment aujourd'hui avoir un objet $a$, être mère, tenir une position de grand Autre, tout en demeurant objet a pour un homme, être une femme en position subjective, c'est-à-dire " se faire " objet cause du désir de l'homme ? Congeler un bébé a cet « avantage » de contourner définitivement la question (du manque), car le tuer tout en le conservant s'oppose au renoncement nécessaire, c'est-à-dire à la perte ; un enfant mort vient ici en place d'un enfant qui symboliserait la perte.

Pour sublimer, aimer, attraper la satisfaction dans le manque et la métaphore, faire semblant de croquer le bébé qui pourra tranquillement se donner à croquer, faire le tour de l'objet et non pas le rejoindre, il faut partir de quelque part, ici le champ pulsionnel de la dévoration. Une " séparation » inarticulée à une " aliénation ", c'est une déchirure qui ne décomplète pas la mère déjà trop castrée et qui meurtrit l'enfant pour qui l'affaire, avant d'être " comprise ", voire avant même d'être "vue ", est déjà " conclue ", trop vite conclue.

Passer de la perte au manque nécessite qu'il y ait d'abord perte, puis symbolisation en manque. Ici, il y a manque et semble aboli le temps du deuil néces-

20. D. W. Winnicott, 1960, "La théorie de la relation parent-nourrisson », dans De la pédiatrie à la psychanalyse, Paris, Payot, 1969.

21. J. Lacan, Le Séminaire, Livre XVII (1969-1970), L'envers de la Psychanalyse, Paris, Le Seuil, 1991, p. 129. 
saire à l'acceptation du manque, ce temps qui engendre la dynamique désirante de chacun.

Dans cette clinique adolescente, des Antigones assignées à une place d'objet pur dans un impossible espace vide se précipitent dans ce vide et il est difficile et long de prendre la parole là où il n'y a plus que de l'acte ou des libidos pulsionnelles.

Si « assignation » relève, en termes juridiques, de l'« affectation d'un fonds au paiement d'une dette », il avait pour sens, en vieil anglais, celui d'un « rendezvous secret romantique $» . .$.

Se pourrait-il que la modification de la structure du désir de la mère ait pour conséquence le ratage d'une rencontre fondamentale et fondatrice pour le bébé avec l'intime maternel ? ${ }^{22}$

\section{RÉSUMÉ}

Ce texte propose une hypothèse relative à la jouissance maternelle postmoderne recelée dans une clinique adolescente de l'acte : la mère pourrait bien, en se présentifiant comme immédiatement barrée, empêcher, en ne pouvant investir libidinalement son enfant, le temps de l'illusion et donc celui de la désillusion. La conséquence de ce défaut de l'instant logique de voir, auquel vient se substituer le moment de conclure, produit un sujet ayant à exister comme divisé sans avoir pris acte de la perte qui le fonde, c'est-à-dire sans avoir traversé le temps pour comprendre. Comme si sa vie commençait par la conclusion... Le sujet existe ici avec un objet perdu, mais dont la perte ne repose pas sur le mythe d'une complétude antérieure.

Mots-CLÉS

Illusion, désillusion, perte, objet, jouissance, adolescence.

\section{SUMMARY}

This paper proposes an hypothesis on the postmodern maternal enjoyment articulated with teen-agers'acting-out : the mother presenting herself as immediately castrated can not invest libidinally her child, the time of illusion/disillusion. The consequence of this absence of the logical time to see which replaces the time to conclude lets a subject having to exist as divided without taking note of the loss which he is based on, that is to say without going through time to understand. As if his life began with the conclusion... The subject is here with a lost object but the loss does not rest on the myth of completeness earlier.

KEY-WORDS

Illusion, disillusion, loss, object, enjoyment, adolescence.

22. Remerciements à Jéremie Salvadero et Elena Argyriadès pour leur relecture de ce texte et les échanges précieux que nous avons pu avoir sur cette hypothèse. 